\title{
FROM THE EDITOR
}

\section{Vulnerability and palliative care}

As an area of clinical focus or academic concern, medicine has paid very little attention to the topic of vulnerability. It does not typically appear on medical curricula, and in standard medical textbooks, such as Harrison's or Lange's, vulnerability is all but absent. Vulnerable plaques are of course covered extensively, but as for vulnerable people, they somehow seem to be missing from the medical radar. Most physicians would argue that vulnerability is a meta-phenomena, a manifestation of illness or debility that derives from a breach or lack of bodily integrity. As such, people who are sick or disabled, those with diminished competence or decisionmaking capacity, are de facto vulnerable, because their bodies and minds do not function normally.

What this "bodily focused" understanding of vulnerability offers by way of simplicity, it fails to deliver by way of depth. For example, how does one define "normal", and do rigidly applied judgments of normality relegate those who fall below this standard to a status of lower quality, lesser worth, or diminished value? This can happen unwittingly and without malign intent, leading to perceptions of suffering and poor quality of life, which serve to heighten vulnerability, when seen through a normal-centric lens.

Disability rights activist and scholar Carol Gill states, "disabled people are not innately vulnerable, but are made so by a social environment that devalues human differences" (Gill, 2006). In other words, people can be made to feel more at risk than others because of the circumstances in which they live and the assumptions that society holds about them. This assertion certainly resonates with the findings and experiences of our research team, funded by the Canadian Institutes of Health Research, to examine vulnerability in end-of-life care. Working with colleagues from the disability community, we discovered that vulnerability is not merely an experience of the unfortunate few, but rather, a universal condition and part of our collective humanity. The Vulnerability Model of Palliative Care that we developed (Stienstra \& Chochinov, 2006) illustrates that the more people are marginalized, stigmatized, and disenfranchised, the more at risk they are of having a narrower range of healthcare options, through to, and including, end-of- life care.

As with most things in life, appropriate responses are predicated on an accurate and honest accounting of circumstances. If vulnerability is, indeed, not simply a state of bodily dysfunction, but a byproduct of bias or social injustice-imposed vulnerability, so to speak-then solutions for its consequences need to be sought on a multitude of fronts. In this special issue of the Palliative \& Supportive Care, the root causes of vulnerability are provided no quarter. But far from being a "finger wagging" exercise, the articles contained herein offer real opportunities to mitigate imposed vulnerability, which so often shapes end-of-life experience.

The article by Chochinov and colleagues examines the application of Dignity Therapy in two distinct samples of the frail elderly; those who have maintained their cognitive capacity and those who have not. It is striking that in both instances, healthcare providers experienced the patient's story-either as told by the patient directly, or by their family member proxy-as a way of shifting their perceptions. After having seen her patient's story-an elderly woman with advanced dementia-the nurse stated, "I see my resident not just how she is now but how she was in her earlier life, who she was, and how she came to be the person she is now." This idea of being able to shift perceptions from the domain of patienthood to the realm of personhood is a core precept of addressing vulnerability and achieving dignity-conserving care.

An article by Stienstra and Chochinov explores barriers to palliative care for those with heightened vulnerability, including those with pre-existing disabilities and impairments, the homeless and the poor; those in conflict with the law, the frail elderly, recent immigrants, and those subjected to discriminatory practices. In these circumstances, vulnerability can be cumulative, with marginalization 
having a cascading effect on peoples' experiences of heightened susceptibility, diminished power, and loss of control. Being treated as outsiders in society, the experiences and needs of these vulnerable populations are not taken into consideration in policy and the design of important systems such as the healthcare system. To redress this intensified vulnerability, the authors call for inclusive palliative care that builds on and extends the strengths of palliative care including a broader unit of care, accessibility and adapting to diversity, dignity-conserving care, and coordination of palliative and other care practices.

In a national Canadian study which examined the interface between disability-related policies and palliative and end-of-life policies, Stienstra and colleagues affirm this point. Their findings illustrate that when these policies fail to align, people with disabilities encounter a multitude of ways in which their vulnerability is heightened or intensified as they face end of life. The critical message is that when policies attend to the dignity, autonomy, and capacity of all individuals, including those who experience intensified vulnerability, vulnerability can be mitigated or lessened. Removing attitudinal, physical, and societal barriers that interfere with appropriate care for some, results in better palliative and end-of-life care for all.

Schwartz and Lutfiyya suggest that the barriers to quality end-of-life care extend well beyond the palliative care ward or hospice, and include "everyday" discourses about what it means to suffer. They suggest that vulnerability is created by common links between suffering, pain, and assisted suicide and that by remaking the ways in which we speak about suffering to a focus on meaning and relationships, we lessen the vulnerability experienced.

Some might perceive, given our focus on vulnerable populations, that this special issue Palliative $\&$ Supportive Care is rather narrow in scope. We would remind you that vulnerability comes from a feeling of being at risk and is part of being human. It is constant and universal, given that all bodies are vulnerable and therefore subject to the uncertainties of being human. For some, vulnerability is a cage, which traps them inside of their bodies and the all too confining perceptions of others. For others, it is a lens, through which differences can be appreciated and diversity embraced. Although it might be too much to ask, perhaps you might want to consider vulnerability as a mirror. In seeing your own reflection, you may discover that, similarly to that of your patients, your path is no less fragile, no less tenuous, and no less uncertain. This humbling realization may be an important step toward being able to provide dignity-conserving palliative end-of-life care.

\section{REFERENCES}

Gill, C.J. (2006). Disability, constructed vulnerability, and socially conscious palliative care. Journal of Palliative Care, 22, 183-191.

Stienstra, D. \& Chochinov, H.M. (2006). Vulnerability, disability and palliative end-of-life care. Journal of Palliative Care, 22, 166-174.

HARVEY MAX CHOCHINOV, M.D. Department of Psychiatry University of Manitoba 3017-675 McDermot Avenue

Winnipeg, $M B$ R3E 0V9 Canada

Email: harvey.chochinov@cancercare.mb.ca

DEBORAH STIENSTRA Disability Studies 128 Education Building University of Manitoba Winnipeg, MB Canada R3T 2N2 Email:d_stienstra@umanitoba.ca 